\title{
Kidney diseases in the time of COVID-19: major challenges to patient care
}

\author{
Hamid Rabb \\ Division of Nephrology, Johns Hopkins University School of Medicine, Baltimore, Maryland, USA.
}

$\mathbf{T}$ he late Colombian Nobel Prize-winning author Gabriel García Márquez wrote "Love in the Time of Cholera," a complex depiction of the struggle to love during an infectious epidemic (1). In the novel, a rational, responsible physician devoted to science is working to eradicate cholera but is pitted against a passionate, emotional, and impulsive man who wins some of the personal battles. Our current battle against the novel coronavirus 2019 disease (COVID-19) caused by SARS-CoV-2 will require both rational medical science as well as passionate and rapid risk-taking. The current COVID-19 pandemic has affected everyone but presents profound consequences for patients with kidney disease, health care providers, and biomedical researchers. In this Viewpoint, I will discuss a number of kidney-specific aspects of COVID-19 infection, noting therapeutic and basic research opportunities.

\section{SARS-CoV-2 entry into kidney epithelial cells via ACE2}

Angiotensin-converting enzyme 2 (ACE2) serves as a receptor for SARS-CoV-1 and SARS-CoV-2 $(2,3)$. ACE2 is expressed in the lung alveolar epithelial cells, oral and nasal mucosa, and other organs, including renal epithelial cells and bladder cells $(4,5)$. Activation of the renin-angiotensinaldosterone system is countered by ACE2. SARS-CoV-2 can bind to renal epithelial cells, injure these cells, and subsequently disrupt whole body fluid, acid-base, and electrolyte homeostasis. By injuring kidney epithelial cells, SARS-CoV-2 also disrupts kidney endocrine production of erythropoietin and vitamin D and impairs blood pressure regulation. Viral entry into renal epithelial cells suggests the possibility that the kidney could also become a viral reservoir once clearance occurs elsewhere and that urine could be an infectious factor. It will be important to determine if other kidney-resident cells, such as podocytes, interstitial cells, and immune cells, become infected. Given widespread use of ACE inhibitors and angiotensin receptor blockers for hypertension and cardiac and renal diseases, it will also be important to determine whether stopping or adding these drugs is beneficial in acute COVID-19 infections (6). At this point, many medical societies have suggested that the health benefits of ACE inhibitors or angiotensin receptor blockers outweigh potential risks in COVID-19, but this is an important unanswered question.

\section{COVID-19 and dialysis}

Over 500,000 patients in the US are currently on dialysis (7). These patients have depressed immune systems, and those using in-center hemodialysis visit densely populated outpatient units three times per week. Patients on kidney dialysis usually have other comorbidities, such as diabetes, hypertension, and cardiac diseases. Rapid responses to COVID-19 by hemodialysis units, which are experienced in limiting the viral spread of HIV, hepatitis B and C, and other pathogens, will decrease but not prevent its spread. Early in the pandemic, COVID-19-positive symptomatic hemodialysis patients were often transferred to hospitals, both to manage their acute illness and to reduce the risk of spread at their dialysis units. With the spread of COVID-19, dedicated outpatient dialysis units will be needed to care for growing numbers of COVID-19-positive stable hemodialysis patients. Increasing the use of home dialysis with home hemodialysis or peritoneal dialysis, already a goal of the US

Conflict of interest: The author has declared that no conflict of interest exists. 
tion agents. Patient with donor-specific antibodies and those undergoing rejection need even higher levels of immunosuppression. Initial data about outcomes of long-term stable kidney transplant patients with COVID-19 infections are still emerging, and it is not clear how these outcomes compare with the general population. However, while acutely lifesaving transplants of heart, lung, and high model for end-stage liver disease (MELD) score livers are continuing, most live donor kidney transplantation is currently on hold. Deceased donor kidney transplantation should probably only be performed when lifesaving, such as for a patient running out of vascular access or a highly sensitized patient who may not come across another compatible donor. When deceased donor organs are being accepted in the resource-limited COVID-19 hospital environment, they will most likely be "higher quality" organs, which will recover quickly from storage and ischemic injury, which goes against the current trend of expanding the organ pool (11). Besides the infection concerns for the fresh transplant patient, the effect of elective transplant procedures on hospital resource utilization, diverting care from critically ill patients with COVID-19, is being factored into decisions to reduce kidney transplantation rates. Newer rapid diagnostic techniques are being evaluated to screen deceased donors for COVID-19 at the organ procurement organization before use as well as to screen recipients on or before arrival for the kidney transplant.

There is also a concern that highly immunosuppressed fresh transplant patients can also be infected during the initial hospitalization or in the community after discharge (12). The role of different immunosuppression agents, such as calcineurin inhibitors and intravenous immunoglobulin, in COVID-19 disease is not established. The current pandemic serves as a reminder to try to carefully reduce immunosuppression in stable patients. When a transplant patient becomes infected, early consideration should be given to discontinue antimetabolite immunosuppression, such as mycophenolate mofetil. There will be an increased opportunity for noninvasive biomarkers, such as genomic tools and donor-derived cell-free DNA, to obviate a patient coming to the hospital for a biopsy $(13,14)$.

\section{COVID-19 and acute kidney injury}

Acute kidney injury (AKI) occurs in about $5 \%$ of hospitalized patients and up to $50 \%$ of patients in the ICU. The mortality rate from AKI is very high, even in the absence of COVID-19 infections. The published incidence of COVID-19-induced AKI is highly variable to date. An early study from China showed a $27 \%$ incidence of AKI in 85 COVID-19-positive patients, as defined by a $30 \%$ decrease in glomerular filtration rate (15). There was an increased risk of AKI associated with age $>60$ years, coexisting hypertension, and coronary artery disease. Six patients had postmortem evaluations that demonstrated severe acute tubular injury, prominent lymphocyte infiltration, detection of viral antigen in tubular epithelial cells, macrophage infiltration, and complement C5b-9 deposition. The lymphocyte and immune cell infiltration found in COVID-19-induced AKI is likely an important pathophysiologic factor in kidney injury and repair $(16,17)$. In another study conducted on 710 hospitalized patients, $44 \%$ of admitted patients had proteinuria, 27\% had hematuria, and 3.2\% had AKI, and these indicators of kidney impairment were associated with high mortality (18). The associated high mortality from AKI, even with availability of dialysis, could be due to deleterious lung-kidney crosstalk during COVID-19 infection and augmentation of inflammation during AKI $(19,20)$. Moving forward, use of sensitive AKI biomarkers beyond just evaluating rises in serum creatinine will aid in early detection of AKI or subclinical kidney injury associated with COVID-19 infections (21).

\section{Future directions}

It is important to recognize the unique challenges facing the community of patients with kidney disease during COVID-19 pandemic. These individuals may be at increased risk of infection or worse outcomes and are already experiencing obstacles in their routine medical care. In addition to the tremendous need and opportunity for clinical and basic research into COVID-19 and kidney diseases, local and international collaborations will be more important than ever. Information coming out in bits and pieces will need to be put into systematic registries, and it will be crucial not to sacrifice scientific rigor in our collective effort to address the new challenges facing patients with kidney diseases in the COVID-19 pandemic.

\section{Acknowledgments}

The author's work is supported by the National Institute of Diabetes and Digestive and Kidney Diseases (grants R01DK104662 and R01DK111209). Chirag Parikh, Daniel C. Brennan, and Sanjeev Noel provided helpful edits.

Address correspondence to: Hamid Rabb, Johns Hopkins Hospital, Ross 965, 720 Rutland Ave., Baltimore, Maryland 21205, USA.Email: hrabb1@jhmi.edu.

1. Márquez GG. Love in the Time of Cholera. Edith Grossman, trans. New York, New York, USA: Alfred A Knopf; 1988.

2. Li W, et al. Angiotensin-converting enzyme 2 is a functional receptor for the SARS coronavirus. Nature. 2003;426(6965):450-454.

3. Hoffmann M, et al. SARS-CoV-2 cell entry depends on ACE2 and TMPRSS2 and is blocked by a clinically proven protease inhibitor [published online March 4, 2020]. Cell. http://doi. org/10.1016/j.cell.2020.02.052.

4. Lin W, et al. Single-cell analysis of ACE2 expression in human kidneys bladders reveals a potential route of 2019-nCoV Infection [preprint]. http://doi.org/10.1101/2020.02.08.939892. Posted on bioRxiv February 18, 2020.

5. Fan C, Li K, Ding Y, Lu WL, Wang J. ACE2 expression in kidney testis may cause kidney testis damage after 2019-nCoV infection [preprint]. http://doi.org/10.1101/2020.02.12.2002 2418. Posted on medRxiv February 13, 2020.

6. Vaduganathan M, Vardeny O, Michel T, McMur ray JJV, Pfeffer MA, Solomon SD. Renin-angiotensin-aldosterone system inhibitors in patients with Covid-19 [published online March 30, 2020]. N Engl J Med. https://doi.org/10.1056/ nejmsr2005760.

7. United States Renal Data System. 2019 USRDS annual data report: Epidemiology of kidney disease in the United States. Bethesda, Maryland, USA: NIH, National Institute of Diabetes and Digestive and Kidney Diseases; 2019. http://www. usrds.org/2019/view/Default.aspx. Accessed April 6, 2020.

8. US Department of Health and Human Services. Advancing American Kidney Health. Washington, DC, USA: US Department of Health and Human Services; 2018. http://aspe.hhs.gov/system/ files/pdf/262046/AdvancingAmericanKidneyHealth.pdf. Accessed April 6, 2020

9. Ma Y, et al. 2019 novel coronavirus disease in hemodialysis (HD) patients: report from one HD center in Wuhan, China [preprint]. http://doi. org/10.1101/2020.02.24.20027201. Posted on medRxiv February 27, 2020.

10. Naicker S, Yang CW, Hwang SJ, Liu BC, Chen JH Jha V. The novel coronavirus 2019 epidemic and 
kidneys [published online March 7, 2020]. Kidney Int. https://doi.org/10.1016/j.kint.2020.03.001.

11. Tullius SG, Rabb H. Improving the supply and quality of deceased-donor organs for transplantation. N EnglJMed. 2018;379(7):693-694.

12. Kumar D, et al. COVID-19: a global transplant perspective on successfully navigating a pandemic [published online March 23, 2020]. Am J Transplant. http://doi.org/10.1111/ajt.15876.

13. Sigdel TK, et al. A urinary Common Rejection Module (uCRM) score for non-invasive kidney transplant monitoring. PLOS ONE. 2019;14(7):e0220052.

14. Bloom RD, et al. Cell-free DNA and active rejection in kidney allografts. J Am Soc Nephrol. 2017;28(7):2221-2232.

15. Diao B, et al. Human kidney is a target for novel severe acute respiratory syndrome coronavirus 2 (SARS-CoV-2) infection [preprint]. http://doi. org/10.1101/2020.03.04.20031120. Posted on medRxiv March 17, 2020.

16. Rabb H, et al. Pathophysiological role of T lymphocytes in renal ischemia-reperfusion injury in mice. Am JPhysiol Renal Physiol. 2000;279(3):F525-F531.

17. D'Alessio FR, Kurzhagen JT, Rabb H. Reparative T lymphocytes in organ injury. JClin Invest. 2019;129(7):2608-2618.

18. Cheng Y, et al. Kidney impairment is associated with in-hospital death of COVID-19 patients [preprint]. http://doi.org/10.1101/2020.02.18.20023 242. Posted on medRxiv February 20, 2020.

19. Kramer AA, Postler G, Salhab KF, Mendez C, Carey LC, Rabb H. Renal ischemia/reperfusion leads to macrophage-mediated increase in pulmonary vascular permeability. Kidney Int . 1999;55(6):2362-2367.

20. Rabb H, et al. Inflammation in AKI: current understanding, key questions, and knowledge gaps. J Am Soc Nephrol. 2016;27(2):371-379.

21. Zhang WR, Parikh CR. Biomarkers of acute and chronic kidney disease. Annu Rev Physiol. 2019;81:309-333. 